\title{
LA GESTIÓN EDUCATIVA EN EL LIDERAZGO DIRECTIVO DE LOS COLEGIOS DEL CIRCUITO 07D01 DEL CANTÓN PASAJE.
}

\section{THE EDUCATIONAL MANAGEMENT IN THE LEADERSHIP LEADERSHIP OF THE SCHOOLS OF THE 07D01 CIRCUIT OF THE PASAJE CANTON.}

\section{Autora: Sandra Maribel Jiménez Riofrio}

E-mail de contacto: sandram.jimrio@gmail.com

Articulo recibido: 30 de Diciembre del 2019

Articulo revisado: 2 de Enero del 2020

Articulo aprobado: 17 de Febrero del 2020

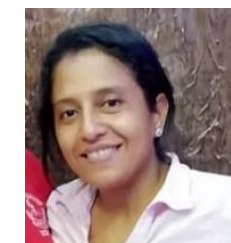

Economista egresada de la Universidad Central del Ecuador (Ecuador) con 5 años de experiencias laboral en la Escuela d Educación Básica Capitán Juan Salinas. Maestrante en la Universidad Nacional Mayor de San Marcos de Lima (Perú), mención Gestión de la Educación.

\section{Resumen}

A nivel de América Latina, la gestión en las instituciones educativas se ha encontrado inmersa a una serie de modificaciones a nivel social con el transcurso del tiempo. Este articulo tiene como objetivo determinar la influencia de la gestión educativa en el liderazgo directivo de los colegios del Circuito 07D01 del cantón Pasaje, provincia de El Oro, en el período lectivo 2017 - 2018. Para ello el tipo de investigación que se utilizará en este articulo será un análisis bibliográfico. Se determino la correlación directa que existe entre las variables de estudio.

Palabras claves: Gestión educativa,liderazgo directivo, análisis bibliográfico.

\begin{abstract}
At the level of Latin America, management in educational institutions has been immersed in a series of changes at the social level over time. This article aims to determine the influence of educational management in the leadership of the schools of the Circuit 07D01 of the Canton Pasaje, province of El Oro, in the 2017-2018 school period. For this, the type of research that will be used in this Article will be a bibliographic analysis. The direct correlation between the study variables was determined.

$\begin{array}{lll}\text { Keywords: } & \begin{array}{l}\text { Educational } \\ \text { managerial } \\ \text { leadership, }\end{array} & \text { bibliographic } \\ \text { analysis. } & & \end{array}$
analysis.
\end{abstract}

\section{Sumário}

No nível da América Latina, a gestão nas instituições de ensino foi imersa em uma série de mudanças no nível social ao longo do tempo. Este artigo tem como objetivo determinar a influência da gestão educacional na liderança das escolas do Circuito 07D01 do Cantão Pasaje, província de El Oro, no período escolar de 2017-2018. Para isso, o tipo de pesquisa que será utilizada neste $\mathrm{O}$ artigo será uma análise bibliográfica. A correlação direta entre as variáveis do estudo foi determinada.

Palavras-chave: Gestão educacional, liderança gerencial, análise bibliográfica

\section{Introducción}

A nivel de América Latina, la gestión en las instituciones educativas se ha encontrado inmersa a una serie de modificaciones a nivel social con el transcurso del tiempo, en donde se puede mencionar que existen cinco factores que inciden de forma significativamente positiva en la calidad de educación, entre ellos se puede señalar: en la década de los años 80 y 90 se ha visto un cambio inminente en la variedad de nuevas reformas acerca de la utilización de recursos en el sistema educativo; el segundo factor hace referencia al apartamiento de la economía, es decir, se ha visto sumergida en una competitividad a nivel mundial; la tercera se trata de la actualización del talento humano a través de capacitaciones, agilidad y nuevas 


\section{Ciencia y Educación (ISSN 2707-3378) \\ Vol. 1 No. 5 \\ Mayo del 2020}

maneras de compromiso; el cuarto factor trata acerca de las adecuaciones en los centros educativos tanto técnicos como sociales; y por último el quinto factor se vincula de forma directa al uso de las TIC en los procesos de enseñanza aprendizaje (Pérez, 2014).

López (2010) en su investigación afirma que hoy en día existen una variedad de estudios tanto nacionales como internaciones acerca de la necesidad urgente de una gestión escolar a través del liderazgo directivo, considerando que las instituciones educativas necesitan con urgencia un cambio a nivel social, cultural y sobre todo profesional en donde mejore la calidad educativa, comprometiéndose de forma general la comunidad educativa (autoridades docentes - representantes legales estudiantes); y de esta manera se logrará buenos resultados en el proceso de enseñanza aprendizaje.

En otros países de América del Sur, se ha divisado que el Ministerio de Educación conocido como Mineduc ha estructurado una variedad de decisiones que están consignadas a iniciar con un nuevo proceso de perfección de la calidad de gestión educativa, en donde el primer involucrado es el director de la institución educativa, ya que se trata de profesionalizar su cargo mejorando su capacidad de líder ante el personal docente y de esa manera convertirse en líder del PEI (Tapia, Becerra, Mansilla, \& Saavedra, 2011).

A nivel nacional, la gestión educativa ha evolucionado de manera positiva mejorando paulatinamente la educación de los establecimientos educativos, considerando que existen 9 Zonas, 140 Distritos y 1200 Circuitos en todo el Ecuador ofertando educación inicial, básica, bachillerato y en algunos de los casos educación para personas adultas y no hay que dejar de lado la inclusión educativa que se da en todos los ámbitos; para esto se ha implementado un nuevo modelo de gestión en donde se ha mejorado la infraestructura, el reclutamiento de nuevos profesionales, adquisición de recursos tanto didácticos como técnicos, con el fin de introducir las TIC en el proceso de enseñanza aprendizaje (Farfán, Mero, \& Sáenz, 2016).

En los colegios del Circuito 07D01 del cantón Pasaje se ha podido evidenciar que existe un nuevo modelo de gestión educativa; sin embargo, esta trata sobre el equipamiento de los centros con recursos y dejando de lado el liderazgo directivo, pues existe una mala administración de parte de quienes ejercer el poder administrativo, considerando que es un punto primordial para fortalecer las relaciones intrapersonales con el personal docente.

\section{Gestión Educativa}

La gestión educativa tiene un tiempo cronológico en su aparición en diferentes países del mundo, en Estados Unidos inició en los años sesenta, en el Reino Unido en los años setenta, mientras que en Latinoamérica en los años ochenta, esto da a notar que su aparición reciente en nuestro país ha provocado que no se conozca a fondo su estructuración y su debida aplicación en las instituciones educativas (Farfán, Mero, \& Sáenz, 2016); por ende es necesario dejar bien en claro en qué consiste este proceso dentro y fuera de los planteles, haciendo un deslinde desde varias perspectivas:

Es imprescindible explicar el concepto de gestión, por cuanto está estrechamente vinculado con la educación, la administración y con la cultura en general, ya que las normas van desde el nivel macro hasta llegar al micro; es decir, las políticas establecidas por los directivos de una institución educativa organizan y controlan los dos elementos básicos 
para el proceso de enseñanza aprendizaje, como es el docente y el alumno (Portugal, 2013).

Según Torres (2015) de acuerdo con su investigación afirma que la gestión está identificada como una visión amplia de las posibilidades reales de una organización para resolver alguna situación o para alcanzar un fin determinado. Siendo un grupo de acciones que se integran entre sí con el fin de cumplir sus objetivos trazados sean estos a corto o a largo plazo.

Por otro lado, Prudencio (2016) manifiesta a través de su estudio que la gestión educativa es como el arte, la ciencia y la tecnología cuyo objeto de estudio es la institución educativa, se caracteriza por ser el elemento configurador de las relaciones demanda-oferta y se concretiza en el sistema de procesos.

Autores como Álvarez, Ibarra y Miranda (2013) han estudiado este concepto mencionando que la gestión educativa está influenciada y mediada por el discurso de las políticas educativas, por sus cambios, retos y exigencias $\mathrm{y}$, por su aplicación en los contextos locales, regionales y nacionales.

De acuerdo con Chacón (2014) la gestión educativa se considera como un sistema de saberes o competencias para la acción, un sistema de prácticas para que las organizaciones realmente puedan optimizar los frutos de su gestión es necesario pasar de una estructura de conocimiento simplista a una estructura de conocimiento compleja.

Por último, Rico (2016) en su investigación sostiene que la gestión educativa no es más que un "proceso organizado y orientado a la optimización de procesos y proyectos internos de las instituciones, con el objetivo de perfeccionar los procedimientos pedagógicos, directivos, comunitarios y administrativos que en ella se movilizan.

En conclusión, la gestión educativa es un modelo organizacional de una estructura que no solo puede guiar una persona sino un conjunto de individuos, con el fin de optimizar los procesos y planes de una institución educativa; además, cabe recalcar que todos los miembros de la comunidad educativa tienen la oportunidad de aportar con una porción de conocimiento para mejorar las operaciones formativas, didácticas, directivas, funcionarias y comunitarias.

La gestión educativa para la transformación de la escuela

Para Farfán, Mero y Sáenz (2016) de acuerdo con su investigación afirman que para que exista una buena gestión educativa el director debe poseer tres funciones que determinen su perfil, entre estas tenemos:

- El director debe poseer un excelente manejo de relaciones interpersonales, es decir, que él como líder de una institución educativa tiene queincentivar la colaboración activa tanto del personal docente como administrativo, con el propósito decumplir con los objetivos, metas y proyectos educativos propuestos.

- El director debe manejar la información suficiente para poder controlar cada una de las gestiones que se le presentan a lo largo del período lectivo, proyectándose un objetivo y una misión que le permita direccionar los proyectos de manera conjunta con los miembros de la comunidad educativa. 
- El director tiene que ser radical en la toma de decisiones para promover nuevos procedimientos, fortalecer el trabajo del personal docente y tiene que disponer de recursos suficientes para ejecutar su función.

Se puede concluir que la gestión educativa es un conjunto de procedimientos que utiliza el directivo para representar como líder a una institución educativa, a través de la orientación y conducción del trabajo del personal docente como administrativo, en donde se trabaja de forma conjunta con cada uno de los miembros de la comunidad educativa, con el propósito de brindar un servicio de calidad en enseñanza y sobre todo en el cumplimiento de sus objetivos y proyectos trazados.

\section{Los desafios de la gestión educativa del siglo $X X I$}

En el siglo XXI se ha divisado nuevos cambios que ha tenido que pasar la gestión educativa que está enfocado a la tendencia del ser humano, es decir, hoy en día las nuevas disposiciones administradoras se centran a la conducción del personal docente, ya que ellos necesitan tener una capacitación y actualización constante para enfrentar los cambios que se han visto en estos últimos diez años exclusivamente de la introducción de las TIC en el proceso de enseñanza aprendizaje (Chacón, 2014).

Además, se puede decir que la gestión educativa no es estandarizada sino más bien depende de las personas que estén en ese cargo, es decir, la comisión directiva de una institución educativa tiene la potestad de manejar a su manera la gestión ya que es influenciada por los factores sociopolíticos que están en el poder y esto varía en las técnicas que se utilice en la evaluación institucional (Prudencio, 2016).

\section{Dimensiones de la gestión educativa}

Para Álvarez, Ibarra y Miranda (2013, pág. 152) existen cuatro dimensiones de la gestión educativa, que se describen a continuación:

La dimensión organizacional. $\mathrm{La}$ comunidad educativa en general (directivo, docentes, estudiantes y representantes legales) están debidamente organizados en torno a reglas y a una normativa que exige la institución educativa como tal, para un buen funcionamiento.

La dimensión pedagógico-didáctica. Esta dimensión hace referencia a las actividades pedagógicas y didácticas que realizan los docentes en el proceso de enseñanza aprendizaje, con el fin de construir el conocimiento de los alumnos afianzado a teorías, modalidades y esquemas.

La dimensión comunitaria. Esta dimensión abarca la participación que tienen cada uno de los actores de la comunidad educativa (directivo, docentes, estudiantes y representantes legales) y la toma de decisiones ante las exigencias de la institución educativa.

La dimensión administrativa. Se trata del análisis que realizan tanto los directivos como el personal docente con referencia al manejo de recursos materiales, tecnológicos, humanos y económicos que sirven para administrar la institución educativa; con el propósito de alcanzar los objetivos planteados en el plantel. 
Modelo Estructural de la Gestión Educativa

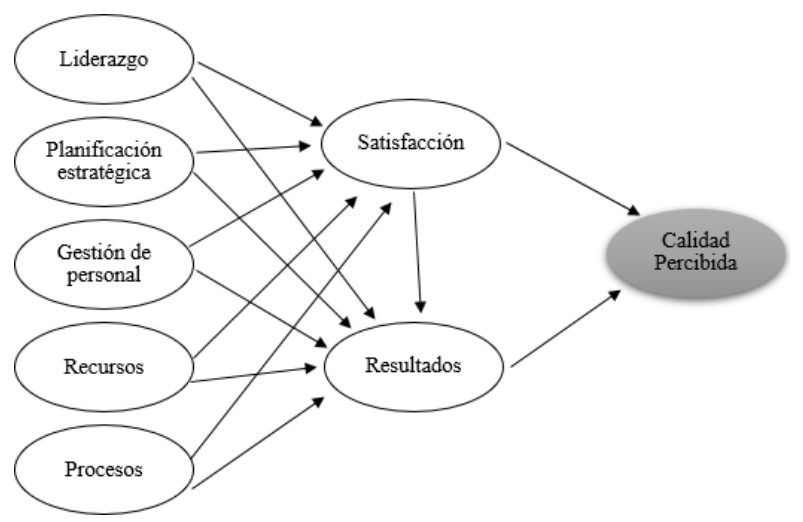

Figura 1: Modelo Estructural de la Gestión Educativa Fuente: La autora

Para López (2006) el modelo estructural de la gestión educativa se combina de 4 variables que están asociadas a los modelos de evaluación y al diagnóstico de la gestión, dando como resultado un modelo propuesto de la gestión educativa. a continuación, se describen operacionalmente cada uno de ellos.

Diagnóstico de la gestión. Esta es la fase inicial en donde los directivos descubren las debilidades y fortalezas que pueden tener las instituciones educativas; y de esta manera se diagnostica la gestión que realizó el director con el personal docente para la mejora de esta; para esto se analiza cada una de las siguientes dimensiones:

- Liderazgo. Es el nivel de perspicaciaque tiene el directivo para gobernar $o$ mandar al personal docente de una institución educativa, con el fin de obtener una gestión de calidad.

- Planificación y estrategia. Es el nivel de perspicacia que tiene el directivo para dirigir los métodos y técnicas utilizados por el personal docente para hacer frente a los proyectos educativos planificados, tomando en consideración a una dirección estratégica.
- Gestión de personal. Es el nivel de perspicacia que tiene el directivo para potenciar las competencias $y$ motivación al personal docente de la institución educativa.

- Recursos. Es el nivel de perspicacia que tiene el directivo para gestionar $\mathrm{y}$ conservar los recursos a utilizar durante el año escolar.

- Procesos. Es el nivel de perspicacia que tiene el directivo para identificar, gestionar, realizar el seguimiento $y$ control de los procesos de la institución educativa.

Satisfacción. Hace referencia al grado de complacencia que tiene el personal docente de la institución educativa, referente a la participación activa en el proceso de enseñanza aprendizaje.

$>$ Resultados. Grado de atribución tanto de los directivos como del personal docente de la institución educativa acerca de los objetivos académicos alcanzados acorde la planificación curricular.

> Calidad Percibida. Grado de atribución tanto de los directivos como del personal docente de la institución educativa acerca de la capacidad de satisfacción de las penurias de los estudiantes y representantes legales, partiendo de los productos recibidos.

\section{El Liderazgo Directivo}

Para López (2010) de acuerdo con su estudio manifiesta que el liderazgo es considerado un:

Compromiso efectivo de los cuadros directivos de la organización y del director general con la calidad, que en la 
práctica significa la definición de los objetivos de calidad por parte del máximo nivel ejecutivo y orientar a toda la organización en ese sentido. (pág. 783)

De acuerdo con Siabato (2013) el liderazgo es:

La habilidad de los directivos y docentes en la gestión del plan estratégico, la gerencia de procesos y en la transformación de la cultura institucional en cuanto al desarrollo de habilidades de participación, comunicación, trabajo en equipo, compromiso con el plan de mejoramiento personal, cultura de la evaluación y mejoramiento en la comunidad educativa. (pág. 40)

Por otro lado, Anderson (2010) afirma que el liderazgo directivo "juega un rol altamente significativo en el desarrollo de cambios en las prácticas docentes, en la calidad de estas prácticas, y en el impacto que presentan sobre la calidad de aprendizaje de los alumnos en las escuelas" (pág. 35).

Tal como se menciona en las citas anteriores, se puede mencionar que el liderazgo es un tema que ha cobrado interés por investigadores en estos últimos años, considerando que ser un líder no es fácil ante los factores que se presentan a lo largo de la administración, pues está fundamentado principalmente en el vínculo que tienen los diferentes autores como es en este caso los miembros de la comunidad educativa.

\section{Teorías de Liderazgo}

De acuerdo con el Centro de Estudios de Políticas y Prácticas en Educación (2009) afirma que hay dos teorías del liderazgo, entre ellas tenemos:
Liderazgo instruccional. Esta teoría hace referencia a la combinación del ambiente y el espacio organizacional que existe en la institución educativa, es decir, en este punto los directores de las instituciones educativas están sumamente preocupados por el proceso de enseñanza aprendizaje tanto de los estudiantes como de su personal docente, ya que influye de forma directa el poder, la jerarquía y el alto conocimiento del director.

Liderazgo transformacional. Por otro lado, el liderazgo transformacional está basado en la formación nuevos líderes, es decir, que el director de la institución educativa se preocupa por el desempeño de su personal docente, motivándolos, concibiendo confianza para que puedan cumplir con sus objetivos; además, este enfoque da la oportunidad que surjan nuevos cambios que engrandecen la contemplación acerca de un liderazgo formativo.

La relación que existe entre la teoría de un liderazgo instruccional y transformacional es que con la fusión de los dos enfoques permite que el director como autoridad máxima de una institución educativa tenga por un lado la potestad de dirigir, controlar y evaluar las actividades que realizan su personal docente en el proceso de enseñanza aprendizaje; sin embargo, no hay que dejar de lado que a más de dirigir estrictamente es necesario que incentive a la comunicación bilateral, elevando el autoconfianza entre los maestros para mejorar sus prácticas docentes.

\section{Estilos de Liderazgo}

Sánchez (2008, págs. 14-20) en su investigación propone cuatro estilos diferentes de liderazgo, que se describen a continuación:

Estilo indiferente. En este grupo seencuentran los directivos con un alto grado de 
permisividad; es decir, que se dejan manipular por las demás personas que tienen más experiencia en el área educativa; además, no se sienten capaces de tomar sus propias decisiones, dejando de lado los controles y mecanismos de las actividades diarias.

Estilo Tecnicista. Este estilo permite que los directivos sean dictadores $\mathrm{u}$ opresores, dirigiendo con un alto liderazgo la toma de decisiones sin tomar en cuenta la opinión de sus demás colaboradores, impartiendo órdenes sin cuestionamiento y sancionando cualquier falta que pudieren cometer, provocando en el personal docente desconfianza, discrepancia y enfado.

Estilo Sociable. Los directivos que se encuentran etiquetados en este tipo de estilo son altamente sociables, es decir, que participan activamente con sus colaboradores, estableciendo en conjunto actividades, tareas y objetivos; además, la toma de decisiones la realizan en consenso, en donde la opinión de cada uno es muy importante a la hora de dirigir el establecimiento educativo; cabe señalar que el directivo aprecia el trabajo en equipo, sin embargo, no deja de lado el desempeño individual de cada uno de sus docentes.

Estilo Sinérgico. Los directivos que se encuentran etiquetados en este tipo de estilo son altamente participativos y sobre todo trabajan en equipo; la toma de decisiones la realizan en consenso, sin embargo, cuando se trata de una persona en específico toma en cuenta su opinión; confían mucho en su personal docente, pues los considera parte esencial del equipo, comunicándose de manera bilateral en ambas direcciones tanto ascendente como descendente; otro puntoimportante es la recompensa, estableciendo incentivos económicos para motivar a sus colaboradores.
Por otro lado, Leal, Albornoz y Rojas (2016) sostienen que una buena gestión educativa depende de forma directa del estilo de liderazgo que tendrá el director de la institución educativa, pues un líder demócrata, interactivo y contribuyente promueve el descubrimiento, la práctica docente y sobre todo permite que fluya un clima organizacional más favorable en su lugar de trabajo.

Por otro lado, Figueroa (2012) en su estudio afirma que existen otros estilos de liderazgo, entre ellos tenemos los siguientes:

Asertivo. Este estilo se refiere a la comprensión que siente el directivo ante el personal docente, presentando medidas precisas para ser controlado con respeto $\mathrm{y}$ responsabilidad, a través de una comunicación abierta.

Punitivo. Este estilo hace referencia a la autoridad que tienen los directivos ante el personal docente, es decir, el ser autoritario permite que los demás sientan temor $\mathrm{y}$ cumplan con sus actividades con un alto grado de presión.

$>$ Inhibicionista. Este estilo permite que los directivos den carta abierta al personal docente para que resuelvan por sí solos los problemas que se les presentan en el procesode enseñanza aprendizaje y les dan pocaatención a los logros alcanzados.

Sobreprotector. Son los directivos que estánal pendiente de todo lo que ocurre en la institución educativa para solucionarlos, ya que piensan que son responsables de todo lo malo o bueno que ocurra en el plantel; además se preocupan de forma excesiva y nodejan que los docentes aprendan de sus equivocaciones.

En conclusión, los diferentes estilos de liderazgo hablan sobre las maneras de ser de loslíderes ya sea en una institución educativa como en una 


\section{Ciencia y Educación (ISSN 2707-3378) \\ Vol. 1 No. 5 \\ Mayo del 2020}

entidad empresarial, puesto que se puede presentar de varias formas y a su vez están relacionadas directamente con el comportamiento que tienen hacia su personal, es decir, el manejo del grupo con el fin de hacer cumplir con el objetivo del establecimiento.

\section{Características del liderazgo directivo}

Para Maureira y Rojas (2013) a través de su investigación afirma que el liderazgo se caracteriza por ser comunicativo, participativo, equitativo y sobre todo motivador, pues de eso depende para sobrellevar a un grupo grande de personas hacia el cumplimiento de los objetivos, metas y proyectos planteados durante un período de tiempo; además, conlleva a una gran responsabilidad, pues de él depende los significativos cambios que puede tener una institución educativa.

De acuerdo con Maureira y Rojas (2013) en su investigación acerca del liderazgo y ladirección, habla sobre las características que tiene el directivo y el líder, citando las siguientes:

El directivo es formal el líder informal. Esto se refiere a la responsabilidad que tienen el directivo ante la institución educativa, que le concede un rango que debe ser respetado por cada uno de los miembros de la comunidad educativa; mientras que el líder no necesita estar en un alto rango para dirigir o controlar las actividades de un plantel.

El directivo tiene autoridad y el líder poder. Esta característica hace referencia a los derechos y obligaciones representado por un cargo ante la institución educativa, fijando normas, políticas y la asignación de trabajo a sus colaboradores; mientras que el líder tiene poder, ganándoselo en base a las actitudes que presenta; es decir, que tiene la capacidadde intervenir de forma directa en las demás personas.
$>$ El directivo influye y el líder inspira. La influencia se trata de injerirse en su equipo de trabajo con la finalidad de conseguir las metas y los objetivos trazados; cuando se habla que el líder inspira se refiere al entusiasmo que emana a sus colaboradores de la institución educativa mediante su destreza para apreciar las emociones de las demás personas.

$>$ El directivo tiene subordinados y el líder, seguidores. Cuando se habla de subordinación se refiere a los colaboradores (personal docente) que tiene el director de una institución educativa que están asignados para cumplir las funciones a su cargo; mientras que el líder tiene seguidores, es decir, personas o un grupo de individuos que trabajan en conjunto persiguiendo un solo objetivo.

$>$ El directivo se hace obedecer y al líder lo siguen voluntariamente. La obediencia que tienen sus colaboradores al director del plantel se debe a la subordinación de cargo; es decir, que tienen que cumplir a cabalidad las órdenes de su jefe para reflejar un adecuado clima laboral; sin embargo, un líder tiene seguidores de manera voluntaria, ya que ha generado la confianza suficiente para trabajar en equipo.

Dadas estas características se puede apreciar que los directivos no solo preceden de autoridady mandatariedad, sino también deben ser líderesen su equipo de trabajo, para dirigir, controlar y evaluar las actividades, tareas y objetivos trazados, manteniendo el bienestar común entre los miembros de la comunidad educativa; además, es preciso recalcar que el director de una institución educativa sea un líder ante el personal docente.

Por otro lado, Martins, Cammaroto, Neris y Canelón (2009) manifiestan que una de las 


\section{Ciencia y Educación (ISSN 2707-3378) \\ Vol. 1 No. 5 \\ Mayo del 2020}

principales características de los directivos es plantearse metas y objetivos y luego cumplirlos a cabalidad, esto demuestra su poder dedecisión para la resolución de problemas que se le presentan a lo largo de su trayectoria en una institución educativa; además, son personas que valoran el esfuerzo de los demás poniéndose en su zapatos, pues es la única manera de vivenciar sus molestias y por último interactúa de forma positiva con sus seguidores demostrándoles empatía.

\section{Elementos del liderazgo directivo}

Para Garbanzo y Orozco (2010) de acuerdo con su estudio el liderazgo directivo tiene una serie de elementos, que se describen a continuación:

Formular objetivos fijos y tener una visión clara.

$>$ Construir un clima laboral adecuado que beneficie el cumplimiento de los objetivos trazados.

Controlar, inspeccionar y evaluar el desempeño del personal docente.

$>$ Servir como ejemplo para sus demás colaboradores.

$>$ Incentivar las acciones del personal docente.

Alto nivel de comunicación entre sus colaboradores.

$>$ Dar mayor importancia a las acciones de sus colaboradores.

> Dar prioridad a las políticas establecidas para el bienestar de la comunidadeducativa.

> Solucionar de forma eficaz los problemas, dejando en segundo plano los culpables.

> Compartir con sus colaboradores la toma de decisiones.

> Formar lazos de amistad con el personal docente.

Atenuando los elementos antes descritos se puede decir que ser un líder conlleva una serie de características que le permiten contar con medios para formar una gestión educativa de manera estratégica, pues tiene la capacidad de cumplir con sus proyectos propuestos y no solo él como director de un establecimiento, sino que lo realiza en grupo, es decir, en conjunto con los miembros de la comunidad educativa, ya que todos conforman a una institución educativa.

\section{Conclusión}

El liderazgo está emparentado al progreso de una habilidad para el beneficio de un enfoque y la gestión apremia a la realización de esta técnica o método lo más eficientemente viable, por lo tanto, al estribar el transcurso del beneficio de la visión organizacional. (López, 2010). Es necesario determinar si el liderazgo tiene una estrecha relación con las estrategias utilizadas por las autoridades de la institución educativa; mientras que la gestión está íntimamente ligada con el sustento del movimiento de un procedimiento, convirtiéndolo en efectivo la ejecución de sus actividades planificadas, proseguida por una distribución organizativa y con ocupaciones funcionarias como la planificación, el control y la organización (López, 2010).

La máxima autoridad como líder transformacional, tiene que obedecer al lineamiento de habilidades y destrezas en el área de la comunicación, con el propósito de interactuar de manera permanente con la colectividad en general, considerando que en la actualidad existen diversos cambios en elámbito social, político, ético, moral y cultural; colando al director en la jerarquía mayor, ya quees quien sirve como mediador para alcanzar la regularización de los cambios mediante la escuela (Martins, Cammaroto, Neris, \&Canelón, 2009). 


\section{Referencias Bibliográficas}

Álvarez, I., Ibarra, M. G., \& Miranda, E. (2013). La Gestión Educativa como Factor decalidad en una Universidad Intercultural. Revista Redalyc: Ra Ximhai, 9(4), 149-156. Obtenido de http://www.redalyc.org/pdf/461/461290040 $\underline{14 . p d f}$

Anderson, S. (2010). Liderazgo Directivo: Claves para una Mejor Escuela. Revista Scielo: Psicoperspectivas, 9(2), 34-52. Obtenido de http://www.scielo.cl/pdf/psicop/v9n2/art03. pdf

Centro de Estudios de Políticas y Prácticas en Educación. (2009). Prácticas de Liderazgo directivo y resultados de aprendizaje. Hacia conceptos capaces de guiar la investigación empírica. Revista Redalyc: Iberoamericana sobre Calidad, Eficacia y Cambio en Educación,7(3), 19-33. Obtenido de http://www.redalyc.org/pdf/551/551140630 03.pdf

Chacón, L. (2014). Gestión educativa del siglo XXI: bajo el paradigma emergente de la complejidad. Revista Redalyc: Omnia, 20(2), 150-161. Obtenido de http://www.redalyc.org/pdf/737/737353960 $\underline{06 . p d f}$

Farfán, Á., Mero, O., \& Sáenz, J. (2016). Consideraciones generales acerca de la gestión educativa. Revista Dialnet: Dominio de las Ciencias, 2(4), 179-190. Obtenido de https://dialnet.unirioja.es/servlet/articulo?co $\underline{\operatorname{dig} 0=5802875}$

Figueroa, M. (2012). Principales modelos de liderazgo: su significación en el ámbito universitario. Revista Scielo: Humanidades Médicas, 12(3), 515-530. Obtenido de http://scielo.sld.cu/pdf/hmc/v12n3/hmc1031 2.pdf

Garbanzo, G. M., \& Orozco, V. H. (2010). Liderazgo para una gestión moderna de procesos educativos. Revista Redalyc: Educación, 34(1), 15-29. Obtenido de http://www.redalyc.org/pdf/440/440139610 $\underline{01 . p d f}$

Leal, F., Albornoz, M., \& Rojas, M. I. (2016). Liderazgo directivo y condiciones para la innovación en escuelas chilenas: el que nada hace, nada teme. Revista Scielo: Estudios pedagógicos, 42(2), 193-205. Obtenido de http://www.scielo.cl/pdf/estped/v42n2/art11.p $\underline{\mathrm{df}}$

López, P. (2006). La gestión como factor de calidad educativa: hacia un modelo causal. Revista Dialnet: Foro educacional (10), 107-132. Obtenido de https://dialnet.unirioja.es/servlet/articulo?co $\underline{\text { digo }=2292741}$

López, P. (2010). El componente liderazgo en la validación de un modelo de gestión escolar hacia la calidad. Revista Scielo: Educação e Pesquisa, 36(3), 779-794. Obtenido de http://www.scielo.br/pdf/ep/v36n3/v36n3a0 9.pdf

Martins, F., Cammaroto, A., Neris, L., \& Canelón, E. (2009). Liderazgo transformacional y gestión educativa en contextos decentralizados. Revista Redalyc: Actualidades Investigativas en Educación, 9(2), 1-27. Obtenido de http://www.redalyc.org/html/447/44713058 008/

Maureira, Ó., \& Rojas, A. (2013). Características del Liderazgo Transformational en Docentes de Lenguaje y Comunicación en una Muestra de Establecimientos Escolares de Vulnerabilidad Social. Revista Scielo: Estudios pedagógicos, 39(1), 115-127. Obtenido de http://www.scielo.cl/pdf/estped/v39n1/art07.p $\underline{\mathrm{df}}$

Pérez, A. (2014). Enfoques de la gestión escolar: una aproximación desde el contexto latinoamericano. Revista Scielo: Educ Educ, 17(2), 357-369. Obtenido de http://www.redalyc.org/pdf/834/834323620 09.pdf 
Portugal, J. (2013). La Gestión Educativa: Una Visión hacia la Formación Docente. Revista Dialnet: Motricidad y Persona (12), 33-40. Obtenido de https://dialnet.unirioja.es/descarga/articulo/ 4735522.pdf

Prudencio, J. (2016). Propuesta de un modelo de gestión educativa relacional compleja. Revista Scielo: Acta Nova, 7(3), 352-366. Obtenido de http://www.revistasbolivianas.org.bo/scielo. php?pid=S168307892016000100009\&script =sci_arttext

Rico, A. D. (2016). La gestión educativa: Hacia la optimización de la formación docente en la educación superior en Colombia. Revista Redalyc: Sophia, 12(1), 55-70. Obtenido de http://www.redalyc.org/pdf/4137/41374464 8005.pdf

Sánchez, I. D. (2008). Los estilos de dirección y liderazgo. Propuesta de un modelo de caracterización y análisis. Revista Redalyc: Pensamiento \& Gestión (25), 1-39. Obtenido de

http://www.redalyc.org/pdf/646/646122410 $\underline{02 . p d f}$

Siabato, M. (2013). Gestión integral y desarrollo educativo. Revista Dialnet: Cooperativismo \& Desarrollo, 21(3), 35-48. Obtenido de https://dialnet.unirioja.es/descarga/articulo/ 4866629.pdf

Tapia, C., Becerra, S., Mansilla, J., \& Saavedra, J. (2011). Liderazgo de los directivos docentes en contextos vulnerables. Revista Scielo: Educ. Educ., 14(2), 389-409. Obtenido de http://www.scielo.org.co/pdf/eded/v14n2/v1 $\underline{4 \mathrm{n} 2 \mathrm{a} 09 . p d f}$

Torres, E. (2015). Gestión educativa y su relación con la práctica docente en las instituciones educativas emblemáticas de la ciudad de Puno - 2014 - Perú. Revista Scielo: Comunicación, 6(1), 56-64. Obtenido de http://www.scielo.org.pe/pdf/comunica/v6n 1/a06v6n1.pdf

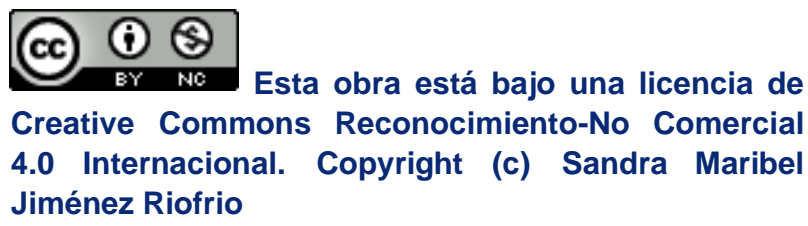
Creative Commons Reconocimiento-No Comercial 4.0 Internacional. Copyright (c) Sandra Maribel Jiménez Riofrio 